\section{(6) OPEN ACCESS}

\title{
Vonoprazan prevents low-dose aspirin-associated ulcer recurrence: randomised phase 3 study
}

\author{
Takashi Kawai, ${ }^{1}$ Kazunori Oda, ${ }^{2}$ Nobuo Funao, ${ }^{2}$ Akira Nishimura, ${ }^{2}$ Yasushi Matsumoto, ${ }^{3}$ \\ Yuji Mizokami, ${ }^{4}$ Kiyoshi Ashida, ${ }^{5}$ Kentaro Sugano ${ }^{6}$
}

\begin{abstract}
- Additional material is published online only. To view please visit the journal online (http://dx.doi.org/10.1136/ gutjnl-2017-314852).

${ }^{1}$ Endoscopy Center, Tokyo Medical University Hospital, Tokyo, Japan

${ }^{2}$ Takeda Development Center, Takeda Pharmaceutical Company Ltd, Osaka, Japan ${ }^{3}$ Department of

Neuroendovascular Therapy, Kohnan Hospital, Sendai, Miyagi, Japan

${ }^{4}$ Endoscopic Center, University of Tsukuba Hospital, Tsukuba, Ibaraki, Japan

${ }^{5}$ Department of

Gastroenterology, Otowa

Hospital, Kyoto, Japan

${ }^{6}$ Department of Medicine, Division of Gastroenterology Jichi Medical University, Tochigi, Japan
\end{abstract}

\section{Correspondence to} Professor Takashi Kawai, Endoscopy Center, Tokyo Medical University Hospital, Tokyo 160-0023, Japan; tkawai@tokyo-med.ac.jp

Received 13 July 2017 Revised 22 October 2017 Accepted 5 November 2017 Published Online First 1 December 2017
ABSTRACT
Objective

Objective Compare efficacy and safety of vonoprazan and lansoprazole for secondary prevention of low-dose aspirin (LDA)-associated peptic ulcers in a 24-week study and long-term extension therapy in separate study.

Design Double-blind, randomised, non-inferiority study; single-blind extension study at 104 Japanese sites, including 621 patients (439 in extension) with a history of peptic ulcers who required long-term LDA therapy. Randomised (1:1:1, computer generated) patients received lansoprazole $15 \mathrm{mg}(\mathrm{n}=217)$, vonoprazan $10 \mathrm{mg}$ ( $n=202)$ or vonoprazan $20 \mathrm{mg}(n=202)$ once daily for 24 weeks (double blind) and $\leq 2$ years (extension). The following measurements were made: 24-week (primary outcome; double blind) and 12-week peptic ulcer recurrence rate, 24-week GI bleeding rate, cumulative incidences of peptic ulcer recurrence and GI bleeding, treatment-emergent adverse events, laboratory results, serum gastrin and pepsinogen I/II concentrations. Results The 24-week peptic ulcer recurrence rate was $2.8 \%, 0.5 \%$ and $1.5 \%$ in the lansoprazole $15 \mathrm{mg}$, vonoprazan $10 \mathrm{mg}$ and vonoprazan $20 \mathrm{mg}$ groups, respectively. Vonoprazan was non-inferior (Farrington and Manning test: margin $8.7 \%$, significance level 2.5\%) to lansoprazole. In the post hoc analyses of the extension study, peptic ulcer recurrence rates were significantly lower with vonoprazan $10 \mathrm{mg}$ (log-rank test, $\mathrm{P}=0.039$ ), but not vonoprazan $20 \mathrm{mg}(\mathrm{P}=0.260)$, compared with lansoprazole $15 \mathrm{mg}$. Gl bleeding rates were higher with lansoprazole compared with two doses of vonoprazan in both 24-week study and extension study.

Conclusion Vonoprazan (10 and $20 \mathrm{mg}$ ) was as effective as lansoprazole $(15 \mathrm{mg})$ in preventing peptic ulcer recurrence during LDA therapy, had a similar long -term safety profile and was well tolerated.

Trial registration numbers NCT01452763; NCT01456247.

\section{INTRODUCTION}

Low-dose aspirin (LDA) is commonly used to prevent the formation of thrombi in patients with ischaemic heart or cerebrovascular diseases. Even at low doses, however, aspirin can cause GI mucosal injury by inhibiting the biosynthesis of intrinsic prostaglandin. ${ }^{1}$ The discontinuation of LDA can increase the risk of cerebral/cardiovascular events. $^{2-4}$ The guideline recommended that LDA be continued in patients with a risk factor of those events $^{5}$; other guidelines have made similar suggestions $^{67}$; however, continuing LDA may contribute

\section{Significance of this study}

What is already known on this subject?

- Due to the increase of cardiovascular diseases with the ageing population, the frequency of antithrombotic prescriptions, including aspirin, is increasing, thereby leading to an elevated risk of GI bleeding.

- Since temporary cessation of antithrombotics increases the risk of mortality, ulcer prevention is important during aspirin therapy.

- Proton pump inhibitor (PPI) is recommended for the prevention of aspirin-related GI adverse events (AEs); however, several days are necessary to achieve a maximal effect and the effect varies depending on the genetic type of CYP2C19 genes.

What are the new findings?

- Based on the different modes of action of vonoprazan compared with conventional PP and its high level antacid effect, vonoprazan showed equivalent efficacy compared with lansoprazole.

- Long-term administration of vonoprazan is well tolerated, and $10 \mathrm{mg}$ can be recommended as a clinical dose.

How might it impact on clinical practice in the foreseeable future?

- Vonoprazan is effective for preventing low-dose aspirin-related GI AEs with stable effects, and it can be considered as a new treatment option.

to increasing the chances of rebleeding. Therefore, it is clinically important to prevent mucosal injury while continuing administration of LDA.

Although proton pump inhibitors (PPIs) are indicated for prevention of gastric or duodenal (peptic) ulcer recurrence during LDA administration, some patients experience ulcer recurrence, ${ }^{89}$ indicating that secondary prevention cannot be completely achieved.

Vonoprazan is a novel potassium-competitive acid blocker developed by Takeda Pharmaceutical Company. Vonoprazan inhibits $\mathrm{H}^{+}, \mathrm{K}^{+}$-ATPase in gastric parietal cells at the final stage of the acid secretory pathway in a $\mathrm{K}^{+}$-competitive and reversible manner. ${ }^{1011}$ From a clinical perspective, vonoprazan has a number of advantages over PPIs. For 
example, vonoprazan does not require an acidic environment for activation and is acid stable, ${ }^{12}$ eliminating the need for an enteric-coated formulation. Whereas PPIs require approximately 3-5 days to achieve maximal inhibition of gastric $\mathrm{H}^{+}, \mathrm{K}^{+}$-ATPase, ${ }^{13}$ vonoprazan exerts a near-maximum inhibitory effect from the first dose and remains effective for 24 hours $^{14}{ }^{15}$. Vonoprazan provides potent and long-lasting inhibition of gastric acid secretion, ${ }^{10} 11{ }^{14-16}$ and its efficacy is therefore expected to be superior to that of PPIs. In a phase 2 study, vonoprazan $(5-40 \mathrm{mg}$ once daily) was highly effective and non-inferior to lansoprazole $30 \mathrm{mg}$ for the treatment of erosive oesophagitis. ${ }^{17}$ However, there have been no studies comparing the efficacy of vonoprazan and PPIs for secondary prevention of LDA-induced ulcers in patients with a history of peptic ulcer.

Against this background, a randomised, double-blind, non-inferiority phase 3 study with single-blind extension study was conducted to investigate the efficacy and safety of vonoprazan for prevention of ulcer recurrence in patients with a history of peptic ulcer who required long-term LDA therapy for cardiovascular and cerebrovascular protection.

\section{MATERIALS AND METHODS Design overview}

This was a multicenter, randomised, double-blind, doubledummy, active-controlled, parallel-group, non-inferiority phase 3 study to compare the efficacy and safety of vonoprazan and lansoprazole for the prevention of recurrent peptic ulcers in patients who required long-term LDA therapy. The 24-week study was conducted between October 2011 and April 2013 at 104 medical institutions across Japan. The double-blind study was followed by a single-blind, parallel-group extension study in which consenting patients continued lansoprazole or vonoprazan for up to 2 years to evaluate safety and efficacy during long-term treatment. The extension study was conducted between March 2012 and October 2013 at 99 of the original study sites. All patients provided written informed consent to participate.

\section{Participants}

Patients were enrolled if they met the following criteria: male or female outpatients $\geq 20$ years of age, endoscopically confirmed history of peptic ulcer before study start and required long-term LDA therapy during the treatment period to prevent the formation of thrombi related to chronic disease (eg, ischaemic heart or cerebrovascular disease).

Patients were excluded if they had: active peptic ulcer or bleeding endoscopically confirmed at the screening visit, current or past history of aspirin-induced asthma, history of surgery or a planned operation affecting gastric acid secretion (eg, upper GI tract resection, vagotomy), current or past history of Zollinger-Ellison syndrome or other gastric acid hypersecretion disorders, clinically significant liver or kidney disorder, including liver tests demonstrating aspartate aminotransferase/alanine aminotransferase values $>2.5$ times the upper limit of normal (ULN), total bilirubin $>2.0$ times the ULN or creatinine levels $>2.0 \mathrm{mg} / \mathrm{dL}$ or history of malignancy within 5 years before screening.

\section{Randomisation and intervention}

The $15 \mathrm{mg}$ dose of lansoprazole was selected as in Japan that is the only dose which is approved for prevention of LDA-induced ulcer recurrence.

Vonoprazan doses of $10 \mathrm{mg}$ and $20 \mathrm{mg}$ were selected based on results from a dose-ranging study conducted in Japanese patients with erosive oesophagitis where the non-inferiority of vonoprazan (at doses of 5, 10, 20 and $40 \mathrm{mg}$ ) to lansoprazole $30 \mathrm{mg}$ was demonstrated. ${ }^{17}$

Patients were randomly assigned to receive lansoprazole $15 \mathrm{mg}$, vonoprazan $10 \mathrm{mg}$ or vonoprazan $20 \mathrm{mg}$ orally once daily in a 1:1:1 ratio according to a unique computer-generated number sequence for study medication, which was preassigned to each study site before the start of treatment. A double-dummy method, using matching vonoprazan placebo and lansoprazole placebo, was used to maintain blinding and ensure that all patients followed the same treatment regimen. Administration of LDA at a dose of $81-324 \mathrm{mg} /$ day was to have been started by the start of treatment. After termination of the double-blind phase, patients were invited to participate in the extension phase during which they continued to receive the same study medication in single-blind fashion for up to 2 years.

\section{Outcomes and measurements}

The primary endpoint of the double-blind study was the rate of peptic ulcer recurrence during the 24-week treatment period. Ulcer was defined as a mucosal defect with white coat of $3 \mathrm{~mm}$ or large, measured using endoscopic forceps. The secondary endpoints were the rate of peptic ulcer recurrence within 12 weeks, the rate of occurrence of bleeding in the stomach or duodenum (based on modified Forrest classification, ${ }^{18}$ cases classified as I or II were reported as bleedings) and the time to peptic ulcer recurrence. Endoscopic findings (for ulcer scarring and recurrence) were investigated independently and blindly by Central Evaluation Committee consisting of two endoscopists.

Safety was assessed on the basis of treatment-emergent adverse events (TEAEs), laboratory test results, ECG findings, vital signs, serum gastrin concentrations and serum pepsinogen I and II concentrations by RI/PEG method. CYP2C19 gene typing were determined by using InvaderPlus (Hologic, Marlborough, Massachusetts, USA)

In the extension study, the primary endpoint was the incidence of TEAEs. Safety was assessed in the same manner as in the double-blind study. The secondary efficacy endpoint was the rate of peptic ulcer recurrence. Additional secondary endpoints were the rate of occurrence of bleeding in the stomach or duodenum and time to peptic ulcer recurrence. The time to bleeding occurrence was defined as a post hoc endpoint.

\section{Follow-up procedures}

Follow-up endoscopy was scheduled at 12 and 24 weeks of treatment. Non-scheduled endoscopy was performed if patients were suspected of having symptoms associated with ulcer onset. Every 4 weeks, clinical laboratory tests (chemistry, haematology and urinalysis) were performed, vital signs were checked, serum gastrin and pepsinogen I/II levels were measured and adherence checks were conducted. Adverse events were monitored continuously throughout the study.

\section{Statistical analysis}

A previous phase 3 study evaluating lansoprazole for prevention of ulcer recurrence during LDA therapy in Japanese subjects reported that the proportion of patients with recurrent peptic ulcer during the 24 -week treatment period was $1.8 \%{ }^{8}$; this same proportion was assumed for lansoprazole and vonoprazan in the current study. Sample size was estimated to ensure a statistical power of $90 \%$ to detect non-inferiority between each vonoprazan group and the lansoprazole group using a Farrington and Manning non-inferiority test ${ }^{19}$ with a non-inferiority margin of $8.7 \%$ (half the value of the treatment difference between 
lansoprazole $15 \mathrm{mg}$ and gefarnate observed in the above-mentioned phase 3 study $^{8}$ ). Assuming a dropout rate of $20 \%$ after randomisation, 210 randomised patients per group (630 total patients) were required to detect non-inferiority of vonoprazan to lansoprazole. In the extension study, 450 patients ( 150 per group) were planned for evaluation of safety.

Analyses were conducted on the full analysis set (FAS), defined as all patients who were randomised and received one or more doses of study medication.

For the primary endpoint (proportion of patients with peptic ulcer recurrence during the 24-week treatment period), the non-inferiority of vonoprazan $10 \mathrm{mg}$ and $20 \mathrm{mg}$ to lansoprazole $15 \mathrm{mg}$ was tested using the Farrington and Manning test with a non-inferiority margin of $8.7 \%$ at a significance level of $2.5 \%$. A closed testing procedure was used for multiplicity adjustment in the tests. Superiority could be claimed if the upper limit of the two-sided $95 \%$ CI of the difference between groups was greater than zero. The other tests were performed at a significance level of $5 \%$. In the extension study, the cumulative incidences of peptic ulcer recurrence and occurrences of bleeding in the stomach or duodenum were calculated by the Kaplan-Meier method.

In the safety analysis set (defined as all patients who received study medication), the frequency distribution of TEAEs was provided for each treatment group. For continuous variables, descriptive statistics were used to summarise observed values and changes from baseline by treatment group for each visit. For categorical variables, shift tables were provided for each treatment group showing the number of patients in each category at baseline and at each post-baseline visit.

Analyses were conducted using SAS V.9.2 software.

\section{Role of the funding source}

Takeda Pharmaceutical Company Ltd. (the Sponsor) and its contractor provided all financial and material support for the study design, data collection, data analysis, data interpretation, and preparation and review of manuscripts. The Sponsor was also responsible for consulting with the authors and members of the study group about the study design and study monitoring.

\section{RESULTS}

\section{Study patients}

Of 809 patients screened, 621 patients were randomised to treatment (figure 1); these patients comprise both the FAS and safety analysis set. The remaining 188 patients were excluded mainly for failing to meet the inclusion criteria $(n=152)$ or voluntary withdrawal $(n=24)$. All randomised patients received study medication and 574 patients completed the double-blind study. Of the 47 patients who discontinued the study prematurely, most were because of adverse events $(n=29)$ or voluntary withdrawal $(n=12)$. Among the patients who completed the double-blind study, 439 entered the extension study, which was originally planned to enrol 140 patients in each arm. Out of 439 patients, 405 completed treatment. Among the 34 patients who discontinued treatment $(11,12$ and 11 patients in the lansoprazole $15 \mathrm{mg}$, vonoprazan $10 \mathrm{mg}$ and vonoprazan $20 \mathrm{mg}$ groups, respectively), the main reasons were adverse events $(n=22)$ or voluntary withdrawal $(n=7)$.

There were no clinically meaningful differences among treatment groups in patient characteristics at baseline (table 1). Adherence with study medication was high across all treatment groups (>99\%). The median duration of exposure was approximately 52 weeks (overall range $2-684$ days ( $<1$ to 98 weeks)) in all treatment groups.

\section{Efficacy}

In the FAS population, the proportion of patients with endoscopically confirmed recurrent peptic ulcer during the 24-week treatment period (primary endpoint) was higher in the lansoprazole $15 \mathrm{mg}$ group $(2.8 \%$; 6 of 213 patients) than in the vonoprazan $10 \mathrm{mg}(0.5 \% ; 1$ of 197 patients) and vonoprazan $20 \mathrm{mg}$ groups (1.5\%; 3 of 196 patients). The differences in recurrence rate between the lansoprazole $15 \mathrm{mg}$ group and the vonoprazan $10 \mathrm{mg}$ and $20 \mathrm{mg}$ groups were $-2.3 \%$ (95\% CI -4.743 to $0.124)$ and $-1.3 \%(95 \% \mathrm{CI}-4.095$ to 1.523$)$, respectively. The non-inferiority of vonoprazan to lansoprazole $15 \mathrm{mg}$ was verified (Farrington and Manning test; $\mathrm{P}<0.001$ for each group). The differences in recurrence rates between vonoprazan $10 \mathrm{mg}$ and lansoprazole $15 \mathrm{mg}(-2.3 \%)$ and between vonoprazan $20 \mathrm{mg}$ and lansoprazole $15 \mathrm{mg}(-1.3 \%)$ were not statistically significant for superiority.

The proportions of patients with recurrent peptic ulcer confirmed by endoscopy at 12 weeks (secondary endpoint) were $0.9 \%, 0.5 \%$ and $0.5 \%$ for patients treated with lansoprazole $15 \mathrm{mg}$, vonoprazan $10 \mathrm{mg}$ and vonoprazan $20 \mathrm{mg}$, respectively, and the between-group differences were not statistically significant for superiority. However, the proportion of patients with bleeding in the stomach or duodenum during the 24-week treatment period (secondary endpoint) was significantly higher in the lansoprazole $15 \mathrm{mg}$ group (2.9\%) compared with the vonoprazan $10 \mathrm{mg}(0 \%)$ and vonoprazan $20 \mathrm{mg}(0 \%)$ groups (difference $-2.9 \%$ (95\% CI -5.135 to -0.607 ) for each group).

In exploratory subgroup analyses, the incidence of peptic ulcer recurrence during 24 weeks of treatment with vonoprazan $10 \mathrm{mg}$ or $20 \mathrm{mg}$ was lower than or similar to that with lansoprazole $15 \mathrm{mg}$, regardless of patient Helicobacter pylori status, CYP2C19 genotype, age, smoking status, alcohol consumption status or presence/absence of treatment with other oral antithrombotic drugs (table 2).

In the post hoc analyses of the double-blind study, when the incidence of gastric or duodenal bleeding was stratified by the presence/absence of treatment with oral antithrombotic drugs during the 24-week treatment period, bleeding incidence was higher with oral antithrombotic drugs in lansoprazole $15 \mathrm{mg}$ at $4.4 \%$, but the incidence was $1.7 \%$ without oral antithrombotic drugs. Bleeding incidence among those received oral antithrombotic drugs were $0.0 \%$ in both vonoprazan $10 \mathrm{mg}$ and $20 \mathrm{mg}$ (difference $-4.4 \%$ (95\%CI -8.702 to -0.187$)$ for each group) and the between-group differences were statistically significant for superiority (Wald test, $\mathrm{P}=0.0408$ ). Statistical significance between lansoprazole $15 \mathrm{mg}$ and both vonoprazan $10 \mathrm{mg}$ and $20 \mathrm{mg}$ were observed by Wald test $(\mathrm{P}=0.0408)$.

In the post hoc analyses of the extension study, the cumulative incidence of peptic ulcer recurrence, as calculated by the KaplanMeier method, was significantly lower with vonoprazan $10 \mathrm{mg}$ (log-rank test, $p=0.039)$, but not vonoprazan $20 \mathrm{mg}(\mathrm{p}=0.260)$, compared with lansoprazole $15 \mathrm{mg}$. Ulcer recurrences were limited to gastric ulcers. The cumulative incidence of bleeding in the stomach or duodenum, as calculated by the Kaplan-Meier method (post hoc), was significantly lower with both vonoprazan $10 \mathrm{mg}$ (log-rank test, $\mathrm{p}=0.018$ ) and vonoprazan $20 \mathrm{mg}$ $(\mathrm{p}=0.019)$ compared with lansoprazole $15 \mathrm{mg}$ (figure $2 \mathrm{~A}, \mathrm{~B})$.

\section{Safety}

Across the double-blind and extension studies, the incidences of TEAEs, adverse events for which a causal relationship with study medication could not be eliminated, and adverse events leading to drug discontinuation were similar between treatment 


\section{A. 24-Week Study}

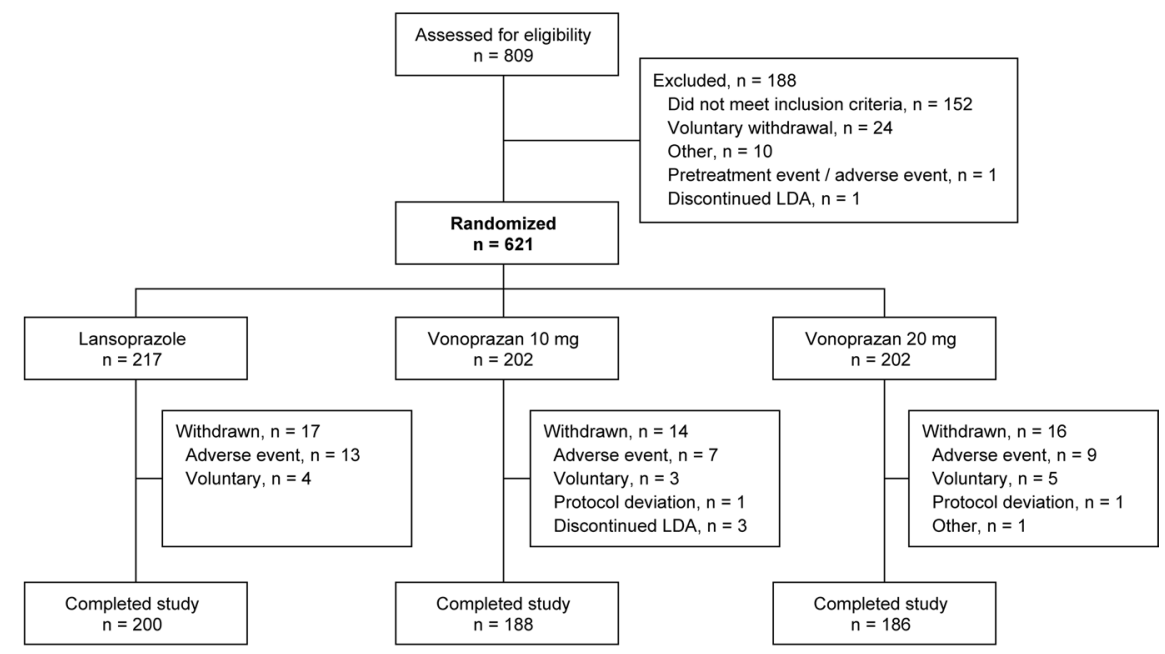

\section{B. Extension Study}

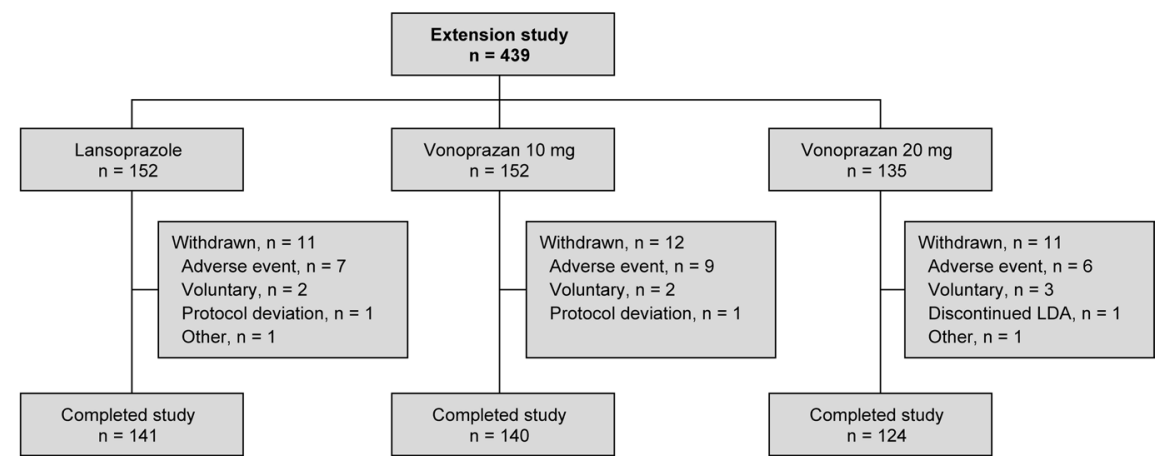

Figure 1 Patient disposition in the double-blind and extension studies. (A) 24-Week study. (B) Extension study. The patients completed the doubleblind study were enrolled consecutively until the enrolments reached the targeted number of patient. By then, the enrolment was ended. LDA, lowdose aspirin.

groups (table 3 ). The number of serious adverse events for which a causal relationship to study medication could not be eliminated was low (1.4\% in the lansoprazole $15 \mathrm{mg}$ group, $2.0 \%$ in the vonoprazan $10 \mathrm{mg}$ and vonoprazan $20 \mathrm{mg}$ groups; table 3$)$. In the lansoprazole $15 \mathrm{mg}$ group, there were three such events in three patients (one case each of acute pancreatitis, cervical adenocarcinoma and acute renal failure). In the vonoprazan $10 \mathrm{mg}$ group, there were five events in four patients (one case each of acute pancreatitis, large intestine polyp, cholelithiasis, thrombotic cerebral infarction and diabetic neuropathy). In the vonoprazan $20 \mathrm{mg}$ group, there were four events in four patients (two cases of gastric cancer and one case each of enterocolitis and oral fibroma). One death due to cardiac failure occurred in the vonoprazan $10 \mathrm{mg}$ group, but this was considered unrelated to study medication.

The most common TEAEs reported in patients (irrespective of causal relationship to study medication) occurred at similar rates in each group and included nasopharyngitis (approximately 30\%) and constipation (approximately 7\%) (table 3). The incidence of diarrhoea was lower in both vonoprazan groups (7.4\%-9.4\%) than in the lansoprazole group (12\%) (table 3). Most adverse events were classified as mild or moderate in intensity $(\geq 98.5 \%$ in each group). The incidence of fracture was similar in all treatment groups. There was no report of Clostridium difficile infection in either the double-blind or extension study (table 3). GI carcinomas were identified in one patient $(0.5 \%)$ treated with lansoprazole, three patients $(1.5 \%)$ treated with vonoprazan $10 \mathrm{mg}$ and six patients (3.0\%) treated with vonoprazan $20 \mathrm{mg}$ (table 3 ). There were no cardiovascular events in the 120 patients who concomitantly received clopidogrel and LDA.

No clinically significant changes were observed in vital signs, haematology tests, serum chemistry, or urinalysis before and after treatment for any group. Serum gastrin levels increased from baseline in all groups after 4 weeks of treatment and increased moderately thereafter up to 52 weeks of treatment (figure 3). Serum gastrin levels were higher with vonoprazan $10 \mathrm{mg}$ and 
Table 1 Patient characteristics at baseline

\begin{tabular}{|c|c|c|c|}
\hline Characteristic & Lansoprazole $15 \mathrm{mg}(\mathrm{n}=217)$ & Vonoprazan $10 \mathrm{mg}(\mathrm{n}=202)$ & Vonoprazan $20 \mathrm{mg}(\mathrm{n}=202)$ \\
\hline Age, mean (SD) years & $68.3(9.06)$ & $68.9(8.02)$ & $69.1(7.18)$ \\
\hline \multicolumn{4}{|l|}{ Gender, n (\%) } \\
\hline Male & $178(82.0)$ & $166(82.2)$ & $163(80.7)$ \\
\hline Female & $39(18.0)$ & $36(17.8)$ & 39 (19.3) \\
\hline BMI, mean (SD) kg/m² & $24.48(3.234)$ & $24.40(3.395)$ & $23.68(3.334)$ \\
\hline Current or ex-smoker, n (\%) & $168(77.4)$ & $158(78.2)$ & $161(79.7)$ \\
\hline Alcohol consumption, n (\%) & $120(55.3)$ & $128(63.4)$ & $118(58.4)$ \\
\hline \multicolumn{4}{|l|}{ Underlying disease, $n(\%)$} \\
\hline Ischaemic heart disease & $136(62.7)$ & $121(59.9)$ & $119(58.9)$ \\
\hline Ischaemic cerebrovascular disorder & $71(32.7)$ & $76(37.6)$ & $80(39.6)$ \\
\hline Other & $39(18.0)$ & $44(21.8)$ & $49(24.3)$ \\
\hline \multicolumn{4}{|l|}{ Helicobacter pylori status, $\mathrm{n}(\%)$} \\
\hline Negative & $127(59.6)$ & $118(58.4)$ & $119(59.2)$ \\
\hline Positive & $86(40.4)$ & $84(41.6)$ & $82(40.8)$ \\
\hline \multicolumn{4}{|l|}{ CYP2C19 polymorphism, $\mathrm{n}(\%)$} \\
\hline Extensive metaboliser & $187(87.0)$ & $163(81.5)$ & $160(79.6)$ \\
\hline Poor metaboliser & $28(13.0)$ & $37(18.5)$ & $41(20.4)$ \\
\hline \multicolumn{4}{|l|}{ Dosage of long-term LDA, $n(\%)$} \\
\hline $100 \mathrm{mg} /$ day (or $81 \mathrm{mg} /$ day aspirin/dialuminate) & $209(96.3)$ & $194(96.0)$ & $193(95.5)$ \\
\hline $200 \mathrm{mg} /$ day (or $162 \mathrm{mg} /$ day aspirin/dialuminate) & $8(3.7)$ & $8(4.0)$ & $9(4.5)$ \\
\hline Other antithrombotic drug ${ }^{*}, \mathrm{n}(\%)$ & $91(41.9)$ & $85(42.1)$ & $81(40.1)$ \\
\hline
\end{tabular}

${ }^{*}$ Clopidogrel, warfarin, ticlopidine or others (additional analysis).

BMI, body mass index; CYP, cytochrome P450; LDA, low-dose aspirin.

$20 \mathrm{mg}$ compared with lansoprazole $15 \mathrm{mg}$, and the degree of increase with vonoprazan was dose dependent. In post hoc analyses of the extension study, $H$. pylori status and ulcer incidence among patients with serum gastrin level of over $500 \mathrm{pg} / \mathrm{mL}$ were comparable in all groups (see Appendix table 1 in the online Supplementary file 1).
Pepsinogen I and II concentrations increased at week 4 in all treatment groups and remained stable through week 52 , as did the pepsinogen I/II ratio (figure 3). The increase of pepsinogen I and II is observed by lansoprazole as well, though the increase was smaller compared with vonoprazan treatments.

Table 2 Incidence of recurrent peptic ulcer during 24 weeks of treatment in subgroups stratified by baseline characteristics

\begin{tabular}{|c|c|c|c|}
\hline \multirow[b]{2}{*}{ Characteristic } & \multicolumn{3}{|c|}{ Recurrence rate, \% (n/N) } \\
\hline & Lansoprazole $15 \mathrm{mg}$ & Vonoprazan $10 \mathrm{mg}$ & Vonoprazan $20 \mathrm{mg}$ \\
\hline \multicolumn{4}{|l|}{ Helicobacter pylori status } \\
\hline Negative & $3.3(4 / 123)$ & $0.9(1 / 114)$ & $2.6(3 / 116)$ \\
\hline Positive & $2.3(2 / 86)$ & $0(0 / 83)$ & $0(0 / 79)$ \\
\hline \multicolumn{4}{|l|}{ CYP2C19 genotype } \\
\hline Extensive metaboliser & $2.7(5 / 184)$ & $0.6(1 / 162)$ & $1.3(2 / 155)$ \\
\hline Poor metaboliser & $3.6(1 / 28)$ & $0(0 / 35)$ & $2.4(1 / 41)$ \\
\hline \multicolumn{4}{|l|}{ Age } \\
\hline$<65$ years & $1.4(1 / 69)$ & $1.9(1 / 53)$ & $0(0 / 50)$ \\
\hline$\geq 65$ to $<75$ years & $2.2(2 / 90)$ & $0(0 / 91)$ & $2.0(2 / 99)$ \\
\hline$\geq 75$ years & $5.6(3 / 54)$ & $0(0 / 53)$ & $2.1(1 / 47)$ \\
\hline \multicolumn{4}{|l|}{ Smoking status } \\
\hline Current or ex-smoker & $1.8(3 / 165)$ & $0.6(1 / 155)$ & $1.9(3 / 155)$ \\
\hline Never smoker & $6.3(3 / 48)$ & $0(0 / 42)$ & $0(0 / 41)$ \\
\hline \multicolumn{4}{|c|}{ Alcohol consumption status } \\
\hline Drinker & $1.7(2 / 119)$ & $0.8(1 / 126)$ & $0.9(1 / 117)$ \\
\hline Never drink & $4.3(4 / 94)$ & $0(0 / 71)$ & $2.5(2 / 79)$ \\
\hline \multicolumn{4}{|l|}{ Oral antithrombotic drug* } \\
\hline Yes & $2.2(2 / 91)$ & $0(0 / 83)$ & $2.5(2 / 79)$ \\
\hline No & $3.3(4 / 122)$ & $0.9(1 / 114)$ & $0.9(1 / 117)$ \\
\hline
\end{tabular}

*Additional analysis.

CYP, cytochrome P450. 

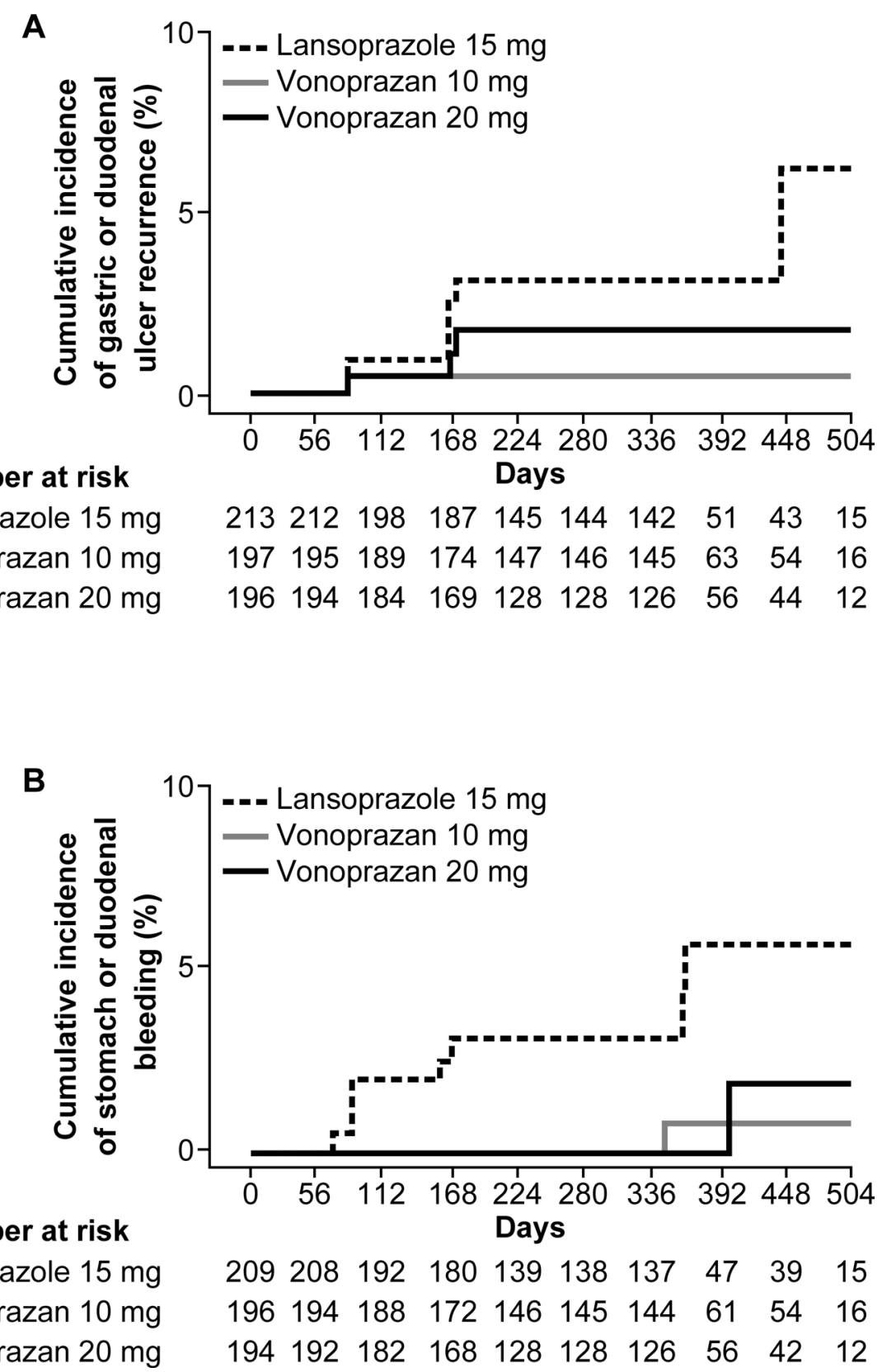

Figure 2 Kaplan-Meier estimates of the cumulative incidence of (A) peptic ulcer recurrence and (B) bleeding in the stomach or duodenum in patients treated with lansoprazole $15 \mathrm{mg}$ (dotted line), vonoprazan $10 \mathrm{mg}$ (solid line) or vonoprazan $20 \mathrm{mg}$ (thick solid line). The numbers of patients at risk for each treatment group are shown in table 1.

\section{DISCUSSION}

This is the first study to assess the safety and efficacy of vonoprazan for the prevention of peptic ulcer recurrence in patients receiving long-term LDA therapy. The double blind, randomised phase of the study demonstrated that vonoprazan $10 \mathrm{mg}$ and $20 \mathrm{mg}$ were non-inferior to lansoprazole $15 \mathrm{mg}$ in the prevention of peptic ulcer recurrence over 24 weeks of treatment. The protective effect of vonoprazan was long lasting, as demonstrated by the low cumulative incidence of both peptic ulcer recurrence and bleeding of the stomach/duodenum during the long-term (up to 2 years) extension study, and there were relatively few safety concerns associated with vonoprazan. Although concern has previously been noted that long-term PPI administration may cause decreased absorption of vitamin B12, calcium and/or magnesium, in our study there was no fluctuation in the serum levels during the prolonged study period. Together, these results indicate that vonoprazan is an effective alternative treatment to PPIs for secondary prevention of peptic ulcer in patients who require LDA therapy for cardiovascular or cerebrovascular protection.

Patients enrolled in this study had a history of peptic ulcer and were receiving long-term LDA therapy to prevent the formation of thrombi; therefore, the patients were at high risk of ulcer recurrence. However, the proportions of patients with recurrent peptic ulcer during 24 weeks of treatment were low in all three groups $(2.8 \%, 0.5 \%$ and $1.5 \%$ in the lansoprazole $15 \mathrm{mg}$, vonoprazan $10 \mathrm{mg}$ and vonoprazan $20 \mathrm{mg}$ groups, respectively), reflecting the efficacy of all three treatment regimens. Non-inferiority of each vonoprazan dose to lansoprazole was verified; however, despite the lower rates of peptic ulcer 
Table 3 Summary of treatment-emergent adverse events (TEAEs) during both the double-blind and extension studies

\begin{tabular}{|c|c|c|c|c|c|c|}
\hline & \multicolumn{2}{|c|}{ Lansoprazole $15 \mathrm{mg}(\mathrm{n}=217)$} & \multicolumn{2}{|c|}{ Vonoprazan $10 \mathrm{mg}(\mathrm{n}=202)$} & \multicolumn{2}{|c|}{ Vonoprazan $20 \mathrm{mg}(\mathrm{n}=202)$} \\
\hline & Events, $\mathbf{n}$ & Patients, n (\%) & Events, $\mathbf{n}$ & Patients, n (\%) & Events, $\mathbf{n}$ & Patients, n (\%) \\
\hline \multicolumn{7}{|l|}{ Summary of TEAEs } \\
\hline Any adverse event & 716 & $184(84.8)$ & 650 & $177(87.6)$ & 637 & $176(87.1)$ \\
\hline Causal relationship to study drug could not be eliminated & 73 & $53(24.4)$ & 45 & $33(16.3)$ & 55 & $39(19.3)$ \\
\hline Leading to study discontinuation & 21 & $20(9.2)$ & 19 & $16(7.9)$ & 15 & $15(7.4)$ \\
\hline Serious adverse events (including deaths) & 39 & $32(14.7)$ & 43 & $33(16.3)$ & 38 & $32(15.8)$ \\
\hline Causal relationship to study drug could not be eliminated & 3 & $3(1.4)$ & 5 & $4(2.0)$ & 4 & $4(2.0)$ \\
\hline Deaths & 0 & 0 & 1 & $1(0.5)$ & 0 & 0 \\
\hline \multicolumn{7}{|l|}{ TEAEs of special interest } \\
\hline Fracture* & 3 & $3(1.4 \%)$ & 7 & $7(3.5 \%)$ & 5 & $5(2.5 \%)$ \\
\hline Clostridium difficile infectiont & 0 & 0 & 0 & 0 & 0 & 0 \\
\hline Gl carcinomał & 1 & $1(0.5 \%)$ & 3 & $3(1.5 \%)$ & 6 & $6(3.0 \%)$ \\
\hline \multicolumn{7}{|l|}{ TEAEs reported by $\geq 5 \%$ of patients in any group } \\
\hline Nasopharyngitis & & $68(31.3)$ & & $60(29.7)$ & & $63(31.2)$ \\
\hline Diarrhoea & & $26(12.0)$ & & $15(7.4)$ & & $19(9.4)$ \\
\hline Constipation & & $16(7.4)$ & & $13(6.4)$ & & $17(8.4)$ \\
\hline Upper respiratory tract inflammation & & $10(4.6)$ & & $12(5.9)$ & & $13(6.4)$ \\
\hline Fall & & $13(6.0)$ & & $11(5.4)$ & & $8(4.0)$ \\
\hline Back pain & & $5(2.3)$ & & $8(4.0)$ & & $16(7.9)$ \\
\hline Elevated creatine phosphokinase & & $10(4.6)$ & & $8(4.0)$ & & $11(5.4)$ \\
\hline Contusion & & $14(6.5)$ & & $7(3.5)$ & & $7(3.5)$ \\
\hline Hypertension & & $7(3.2)$ & & $8(4.0)$ & & $10(5.0)$ \\
\hline Gastroenteritis & & $6(2.8)$ & & $10(5.0)$ & & $8(4.0)$ \\
\hline Eczema & & $7(3.2)$ & & $10(5.0)$ & & $5(2.5)$ \\
\hline
\end{tabular}

*Preferred terms: foot fracture, spinal compression fracture, femur fracture, hand fracture, humerus fracture, lower limb fracture, radius fracture, rib fracture, spinal fracture. †Preferred terms: Clostridia infections, C. difficile bacillaemia, Clostridium colitis, C. difficile colitis, C. difficile infection, C. difficile sepsis, Clostridia test positive, Clostridia enterogastritis, pseudomembranous colitis.

‡Preferred terms: gastric cancer, oesophageal carcinoma, adenocarcinoma gastric.

recurrence in patients receiving vonoprazan, superiority over lansoprazole could not be demonstrated in the 24-week study. During long-term treatment, however, vonoprazan was observed to have lower rates of ulcer recurrence compared with lansoprazole. In addition, both doses of vonoprazan were significantly more effective than lansoprazole at preventing bleeding of the stomach/duodenum during long-term treatment. The results achieved with vonoprazan compare favourably with those reported previously for lansoprazole $15 \mathrm{mg}^{8}$ and are in line with those reported for esomeprazole $20 \mathrm{mg}^{20}$ and rabeprazole $5 \mathrm{mg}$ and $10 \mathrm{mg}^{21}$ in similarly designed studies conducted in similar patent populations.

In addition to oral administration of non-steroidal anti-inflammatory drugs (NSAIDs), confirmed or possible risk factors for ulcer occurrence include history of ulcer, advanced age, $H$. pylori infection, smoking, alcohol intake and concomitant use of anticoagulant therapy. ${ }^{2}$ Patients enrolled in the current study had a confirmed history of ulcer and potentially one or more additional risk factors. For example, the mean age of enrolled patients was approximately 69 years and nearly $80 \%$ were current or ex-smokers. Approximately, 40\% of patients were positive for H. pylori and about $40 \%$ were receiving other antithrombotic drug therapy in addition to LDA. These risk factors could be attributed to the study design using PPI, which is expected to have high efficacy, as an active comparator for the study, unlike previous studies conducted using mucosal-protective drugs. ${ }^{82021}$ Despite these risk factors, analysis of the primary endpoint in patient subgroups defined by baseline characteristics revealed a consistent effect of vonoprazan on ulcer recurrence, with recurrence rates for vonoprazan $10 \mathrm{mg}$ and $20 \mathrm{mg}$ similar to or lower than those for lansoprazole $15 \mathrm{mg}$ within each subgroup. There were studies reporting the GI bleeding incidence rise in proportion to the numbers of antithrombotics prescribed. ${ }^{22} 23$ Therefore, it is of note that in this study, stomach/duodenal bleeding incidence in lansoprazole $15 \mathrm{mg}$ group after 24 weeks was $1.7 \%$ without using antithrombotics with LDA alone, but was $4.4 \%$ with additional antithrombotics use, and increasing tendency was observed in post hoc analyses of the double-blind study although no clinically significant bleeding occurred in the endoscopically confirmed cases. Our study was not designed to evaluate clinically important GI bleeding, therefore these results should be interpreted with caution. However, vonoprazan may offer some advantage on bleeding, such as GI bleeding, which is a harder GI endpoint, considering the significant reduction in endoscopic stigmata of bleeding.

Vonoprazan was well tolerated in the study. During long-term treatment, the safety profiles of the $10 \mathrm{mg}$ and $20 \mathrm{mg}$ doses were similar to that of lansoprazole $15 \mathrm{mg}$. Recent systematic reviews and meta-analyses have suggested a relationship between PPI use and increased risk of fracture ${ }^{24}$ and/or gastroenteritis infections induced by C. difficile. ${ }^{2526}$ In the current study, the incidence of fracture was similar in all three treatment groups and no cases of gastroenteritis infections due to C. difficile were observed.

Long-term use of PPIs has also been associated with a potentially increased risk of GI cancer, possibly related to hypergastrinaemia, although the relationship is questionable. ${ }^{27-30}$ The two patients in $20 \mathrm{mg}$ group who were reported with carcinoma were identified by the Central Evaluation Committee for being diagnosed retrospectively with cancer before the study drug administration. In the study of vonoprazan administered during 
A

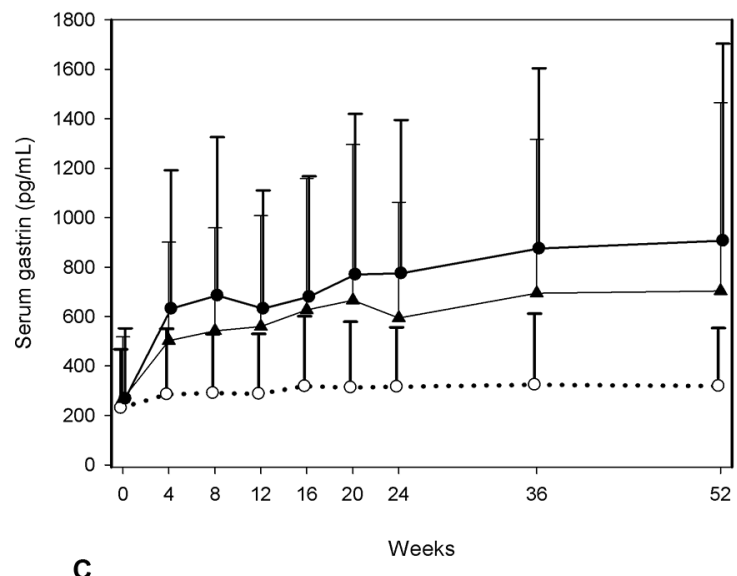

C

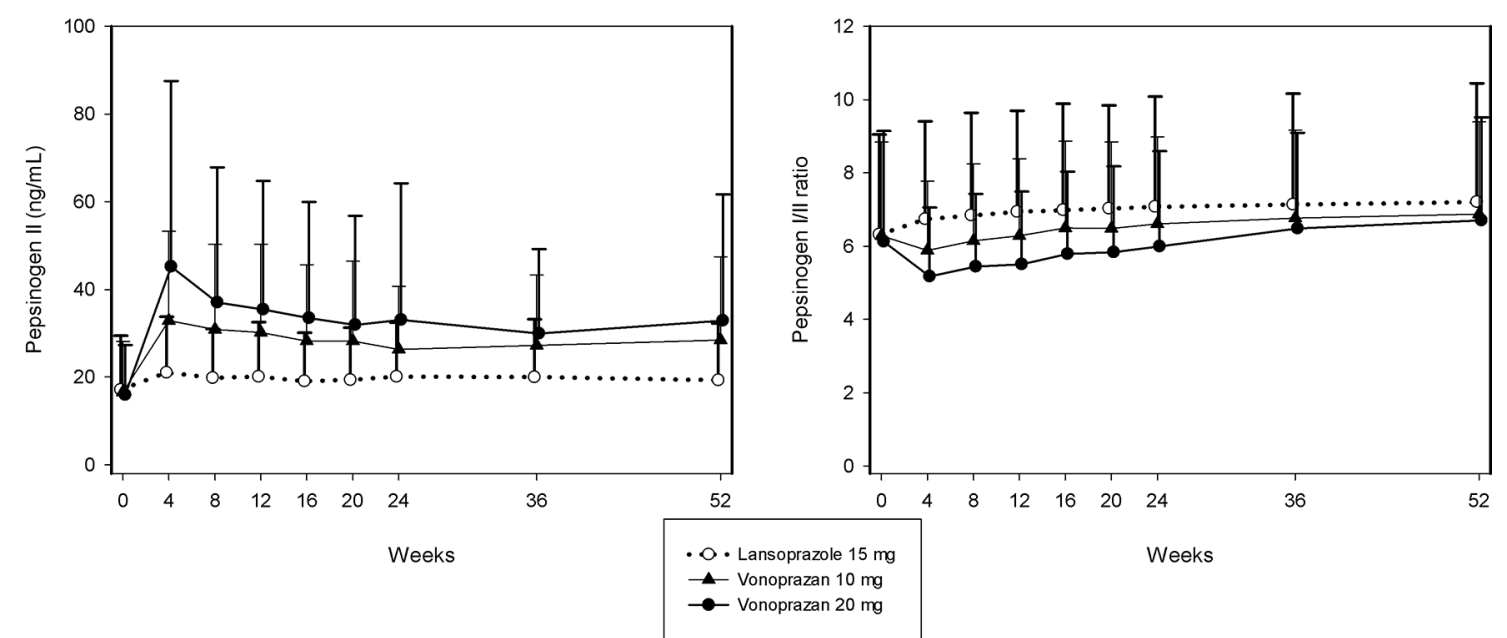

B

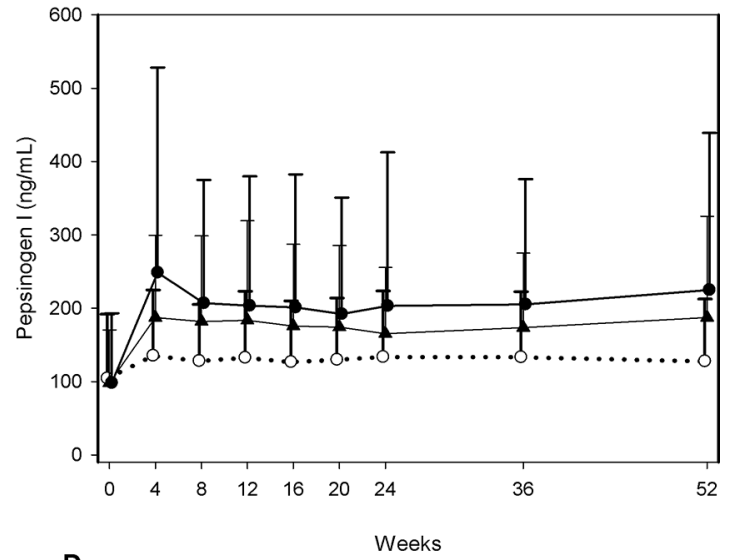

D group). However, the study is somewhat limited by the variable duration of treatment in the extension phase. Also, since the incidence of lower GI adverse events, such as diarrhoea, can be due to a change in intestinal flora, another limitation may be that we did not collect any data on intestinal flora.

In conclusion, vonoprazan at doses of $10 \mathrm{mg}$ and $20 \mathrm{mg}$ once daily was non-inferior to lansoprazole $15 \mathrm{mg}$ for the prevention of ulcer recurrence in Japanese patients with a confirmed history of peptic ulcers who required LDA therapy for cardiovascular or cerebrovascular protection. Vonoprazan was well tolerated and its preventive effect on ulcer recurrence endured with long-term use. In addition, vonoprazan $10 \mathrm{mg}$ and $20 \mathrm{mg}$ showed a significantly greater preventive effect compared with lansoprazole $15 \mathrm{mg}$ on the development of bleeding in the stomach or duodenum. Vonoprazan therefore appears to be a useful alternative to lansoprazole for secondary prevention of LDA-associated peptic ulcers at the recommended clinical daily dose of vonoprazan of $10 \mathrm{mg}$ (no therapeutic advantage in $20 \mathrm{mg}$ dose of vonoprazan over $10 \mathrm{mg}$ was shown).

Acknowledgements The authors would like to thank all contributing members listed in the supplementary appendix 2. The results of this study were previously presented at the Digestive Disease Week (DDW) 2014 and at the Japanese Digestive Disease Week (JDDW) 2014. All authors approved the final version of the article, including the authorship list. Writing assistance was provided by Content Ed Net and by Tania Dickson and Rebecca Lew, CMPP of ProScribe, Envision Pharma Group. a conservative non-inferiority margin and a reliable active control event rate (ie, $2.8 \%$ ulcer recurrence rate in the lansoprazole 
Contributors TK is acting as the guarantor of the article, was the medical expert and was responsible for the study design and for preparation of the manuscript. KO and AN were responsible for the study design and operations. NF was responsible for statistical analysis. YM was the medical advisor for the study. YM and KA were the consultants and clinical endpoint committee members. KS was the coordinating investigator of this study. All coauthors, in addition to having input into the design, conduct or interpretation of the study, critically reviewed the manuscript, and necessary revisions were made to accommodate their suggestions and opinions.

Funding Study and writing assistance was funded by Takeda Pharmaceutical Company Ltd.

Competing interests KO, NF and AN are employees of Takeda Pharmaceutical Company Ltd.

Ethics approval The studies were approved by the Institutional Review Board at each study site and were conducted in accordance with the International Conference on Harmonization Guidelines for Good Clinical Practice, the principles of the World Medical Association Declaration of Helsinki and Japanese regulatory requirements. The studies were registered at ClinicalTrials.gov: NCT01452763 (double blind); NCT01456247 (extension).

Provenance and peer review Not commissioned; externally peer reviewed.

Open Access This is an Open Access article distributed in accordance with the Creative Commons Attribution Non Commercial (CC BY-NC 4.0) license, which permits others to distribute, remix, adapt, build upon this work non-commercially, and license their derivative works on different terms, provided the original work is properly cited and the use is non-commercial. See: http://creativecommons.org/ licenses/by-nc/4.0/

(c) Article author(s) (or their employer(s) unless otherwise stated in the text of the article) 2018. All rights reserved. No commercial use is permitted unless otherwise expressly granted.

\section{REFERENCES}

1 Iwamoto J, Saito Y, Honda A, et al. Clinical features of gastroduodenal injury associated with long-term low-dose aspirin therapy. World J Gastroenterol 2013;19:1673-82.

2 Ferrari E, Benhamou M, Cerboni P, et al. Coronary syndromes following aspirin withdrawal: a special risk for late stent thrombosis. J Am Coll Cardiol 2005;45:456-9.

3 Maulaz AB, Bezerra DC, Michel P, et al. Effect of discontinuing aspirin therapy on the risk of brain ischemic stroke. Arch Neurol 2005;62:1217-20.

4 Sung JJ, Lau JY, Ching JY, et al. Continuation of low-dose aspirin therapy in peptic ulcer bleeding: a randomized trial. Ann Intern Med 2010;152:1-9.

5 Satoh K, Yoshino J, Akamatsu T, et al. Evidence-based clinical practice guidelines for peptic ulcer disease 2015. J Gastroenterol 2016;51:177-94.

6 Laine L, Jensen DM. Management of patients with ulcer bleeding. Am J Gastroenterol 2012;107:345-60.

7 Dworzynski K, Pollit V, Kelsey A, et al. Management of acute upper gastrointestinal bleeding: summary of NICE guidance. BMJ 2012;344:e3412.

8 Sugano K, Matsumoto Y, Itabashi T, et al. Lansoprazole for secondary prevention of gastric or duodenal ulcers associated with long-term low-dose aspirin therapy: results of a prospective, multicenter, double-blind, randomized, double-dummy, activecontrolled trial. J Gastroenterol 2011;46:724-35.

9 Yeomans N, Lanas A, Labenz J, et al. Efficacy of esomeprazole (20 mg once daily) for reducing the risk of gastroduodenal ulcers associated with continuous use of lowdose aspirin. Am J Gastroenterol 2008;103:2465-73.

10 Hori Y, Imanishi A, Matsukawa J, et al. 1-[5-(2-Fluorophenyl)-1-(pyridin-3-ylsulfonyl)$1 \mathrm{H}$-pyrrol-3-yl]-N-methylmethanamine monofumarate (TAK-438), a novel and potent potassium-competitive acid blocker for the treatment of acid-related diseases. J Pharmacol Exp Ther 2010;335:231-8.

11 Shin JM, Inatomi N, Munson K, et al. Characterization of a novel potassiumcompetitive acid blocker of the gastric H,K-ATPase, 1-[5-(2-fluorophenyl)-1-(pyridin-3ylsulfonyl)-1H-pyrrol-3-yll-N-methylmethanamine monofumarate (TAK-438). J Pharmacol Exp Ther 2011;339:412-20.

12 Hori Y, Matsukawa J, Takeuchi T, et al. A study comparing the antisecretory effect of TAK-438, a novel potassium-competitive acid blocker, with lansoprazole in animals. J Pharmacol Exp Ther 2011;337:797-804.
13 Andersson K, Carlsson E. Potassium-competitive acid blockade: a new therapeutic strategy in acid-related diseases. Pharmacol Ther 2005;108:294-307.

14 Sakurai Y, Mori Y, Okamoto H, et al. Acid-inhibitory effects of vonoprazan $20 \mathrm{mg}$ compared with esomeprazole $20 \mathrm{mg}$ or rabeprazole $10 \mathrm{mg}$ in healthy adult male subjects - a randomised open-label cross-over study. Aliment Pharmacol Ther 2015;42:719-30.

15 Sakurai Y, Nishimura A, Kennedy G, et al. Safety, tolerability, pharmacokinetics, and pharmacodynamics of single rising TAK-438 (Vonoprazan) doses in healthy male Japanese/non-Japanese subjects. Clin Trans/ Gastroenterol 2015;6:e94.

16 Matsukawa J, Hori Y, Nishida $\mathrm{H}$, et al. A comparative study on the modes of action of TAK-438, a novel potassium-competitive acid blocker, and lansoprazole in primary cultured rabbit gastric glands. Biochem Pharmacol 2011;81:1145-51.

17 Ashida K, Sakurai Y, Nishimura A, et al. Randomised clinical trial: a dose-ranging study of vonoprazan, a novel potassium-competitive acid blocker, vs. lansoprazole for the treatment of erosive oesophagitis. Aliment Pharmacol Ther 2015;42:685-95.

18 Kohler B, Riemann JF. Upper GI-bleeding - value and consequences of emergency endoscopy and endoscopic treatment. Hepatogastroenterology 1991;38:198-200.

19 Farrington CP, Manning G. Test statistics and sample size formulae for comparative binomial trials with null hypothesis of non-zero risk difference or non-unity relative risk. Stat Med 1990;9:1447-54.

20 Sugano K, Choi MG, Lin JT, et al. Multinational, double-blind, randomised, placebocontrolled, prospective study of esomeprazole in the prevention of recurrent peptic ulcer in low-dose acetylsalicylic acid users: the LAVENDER study. Gut 2014;63:1061-8.

21 Iwakiri R, Higuchi K, Kato M, et al. Randomised clinical trial: prevention of recurrence of peptic ulcers by rabeprazole in patients taking low-dose aspirin. Aliment Pharmacol Ther 2014;40:780-95.

22 Hermosillo AJ, Spinler SA. Aspirin, clopidogrel, and warfarin: is the combination appropriate and effective or inappropriate and too dangerous? Ann Pharmacother 2008;42:790-805.

23 Hansen ML, Sørensen R, Clausen MT, et al. Risk of bleeding with single, dual, or triple therapy with warfarin, aspirin, and clopidogrel in patients with atrial fibrillation. Arch Intern Med 2010;170:1433-41.

24 Yu EW, Bauer SR, Bain PA, et al. Proton pump inhibitors and risk of fractures: a metaanalysis of 11 international studies. Am J Med 2011;124:519-26.

25 Bavishi C, Dupont HL. Systematic review: the use of proton pump inhibitors and increased susceptibility to enteric infection. Aliment Pharmacol Ther 2011;34:1269-81.

26 Deshpande A, Pant C, Pasupuleti V, et al. Association between proton pump inhibitor therapy and Clostridium difficile infection in a meta-analysis. Clin Gastroenterol Hepatol 2012;10:225-33.

27 Ahn JS, Eom CS, Jeon CY, et al. Acid suppressive drugs and gastric cancer: a metaanalysis of observational studies. World J Gastroenterol 2013;19:2560-8.

28 Laine L, Ahnen D, McClain C, et al. Review article: potential gastrointestinal effects of long-term acid suppression with proton pump inhibitors. Aliment Pharmacol Ther 2000;14:651-68

29 Poulsen AH, Christensen S, McLaughlin JK, et al. Proton pump inhibitors and risk of gastric cancer: a population-based cohort study. Br J Cancer 2009;100:1503-7.

30 Thomson AB, Sauve MD, Kassam N, et al. Safety of the long-term use of proton pump inhibitors. World J Gastroenterol 2010;16:2323-30.

31 Mizokami Y, Oda K, Funao N, et al. Vonoprazan prevents ulcer recurrence during long-term NSAID therapy: randomised, lansoprazole-controlled non-inferiority and single-blind extension study. Gut 2018;67:1033-41.

32 Ashida K, Sakurai Y, Hori T, et al. Randomised clinical trial: vonoprazan, a novel potassium-competitive acid blocker, vs. lansoprazole for the healing of erosive oesophagitis. Aliment Pharmacol Ther 2016;43:240-51.

33 Di Mario F, Ingegnoli A, Altavilla N, et al. Influence of antisecretory treatment with proton pump inhibitors on serum pepsinogen I levels. Fundam Clin Pharmacol 2005; 19:497-501.

34 Gilard M, Arnaud B, Cornily JC, et al. Influence of omeprazole on the antiplatelet action of clopidogrel associated with aspirin: the randomized, double-blind OCLA (Omeprazole CLopidogrel Aspirin) study. J Am Coll Cardiol 2008;51:256-60.

35 Lau WC, Gurbel PA. The drug-drug interaction between proton pump inhibitors and clopidogrel. CMAJ 2009;180:699-700.

36 Chitose T, Hokimoto S, Oshima S, et al. Clinical outcomes following coronary stenting in Japanese patients treated with and without proton pump inhibitor. Circ J 2012;76:71-8 